\title{
SUBORDINATE STATISTICAL STRUCTURES
}

UDC 519.21

\author{
Z. K. ZERAKIDZE AND G. A. SOKHADZE
}

\begin{abstract}
General statistical structures are considered in this paper. It is shown that a general statistical structure can be reduced to some orthogonal or absolutely continuous statistical structure.
\end{abstract}

The problems of statistics of stochastic processes usually deal with a family of distributions of an observable trajectory of a stochastic process. It is natural to study the set of all possible trajectories and to associate an arbitrary distribution of the stochastic process with a probability measure in the space of all trajectories. It is important for such an association that all possible distributions have a common support. Note that a common support exists in all natural cases.

If $\mu$ is a $\sigma$-finite measure, then one can construct a probability measure $\widetilde{\mu}$ such that $\mu$ is equivalent to $\widetilde{\mu}$. Indeed, let $(E, S)$ be a measurable space and let $\mu$ be a $\sigma$-finite measure in $S$. Then $E=\sum_{k=1}^{\infty} A_{k}$, where $A_{k}$ are $S$-measurable and disjoint sets and $\mu\left(A_{k}\right)<\infty$ for all $k$. Now we choose a sequence of nonnegative numbers $\rho_{k}$ such that $\sum_{k=1}^{\infty} \rho_{k} \mu\left(A_{k}\right)=1$. Put

$$
\widetilde{\mu}(B)=\sum_{k=1}^{\infty} \rho_{k} \mu\left(A_{k} B\right) .
$$

Then $\mu \sim \widetilde{\mu}$ and $\widetilde{\mu}(E)=1$.

This means that one can transfer the case of $\sigma$-finite measures to the case of equivalent probability measures in finite dimensional spaces. This is not always possible in infinite dimensional spaces.

An exceptional role of absolutely continuous and orthogonal structures is well known in statistics (see, for example, [1, [2]). Below we consider some important cases where the general setting can be reduced to similar problems of estimation of parameters for other absolutely continuous or orthogonal statistical structures. First we discuss some examples.

Example 1. Let $\Theta$ consist of two points $\theta_{1}$ and $\theta_{2}$ and let two probability measures $\mu_{\theta_{1}}$ and $\mu_{\theta_{2}}$ be defined in a measurable space $(E, S)$. The set $E$ can be represented as the union of three disjoint subsets $A_{1}, A_{2}$, and $A_{3}$, that is, $E=A_{1}+A_{2}+A_{3}$, and moreover, $\mu_{\theta_{1}} \sim \mu_{\theta_{2}}$ in the set $A_{2}, \mu_{\theta_{2}}\left(A_{1}\right)=0$, and $\mu_{\theta_{1}}\left(A_{3}\right)=0$.

2010 Mathematics Subject Classification. Primary 62H05, 62H12.

Key words and phrases. Statistical structures, absolute continuity, orthogonality.

The paper is based on the talk presented at the International Conference "Modern Stochastics: Theory and Applications II" held September 7-11, 2010, at Kyiv National Taras Shevchenko University and dedicated to the anniversaries of prominent Ukrainian scientists, Anatoliı Skorokhod, Vladimir Korolyuk, and Igor Kovalenko. 
Consider the following probability measures:

$$
\nu_{1}(A)=\frac{\mu_{\theta_{1}}\left(A \cap A_{1}\right)}{\mu_{\theta_{1}}\left(A_{1}\right)}, \quad \nu_{2}(A)=\frac{\mu_{\theta_{2}}\left(A \cap A_{3}\right)}{\mu_{\theta_{2}}\left(A_{3}\right)}, \quad \nu_{3}(A)=\frac{\mu_{\theta_{1}}\left(A \cap A_{2}\right)}{\mu_{\theta_{1}}\left(A_{2}\right)}
$$

for all sets $A \in S$. Then $\left\{E, S, \nu_{k}, k=1,2,3\right\}$ is an orthogonal statistical structure.

Example 2. Let $\Theta$ consist of two points $\theta_{1}$ and $\theta_{2}$ and let $\mu_{\theta_{1}}$ and $\mu_{\theta_{2}}$ be two probability measures in $(E, S)$. Consider the representation $E=A_{1}+A_{2}+A_{3}$ such that $A_{1} \cap A_{3}=$ $A_{2} \cap A_{3}=0, \mu_{\theta_{2}}\left(A_{1}\right)=0, \mu_{\theta_{1}}\left(A_{3}\right)=0$, and the measure $\mu_{\theta_{1}}$ is equivalent to $\mu_{\theta_{2}}$ in the set $A_{2}$. Consider the following measures:

$$
\nu_{1}(A)=\frac{\mu_{\theta_{1}}\left(A \cap A_{2}\right)}{\mu_{\theta_{1}}\left(A_{2}\right)} \quad \text { and } \quad \nu_{2}(A)=\frac{\mu_{\theta_{2}}\left(A \cap A_{2}\right)}{\mu_{\theta_{2}}\left(A_{2}\right)}
$$

for all sets $A \in S$. Then $\left\{E, S, \nu_{k}, k=1,2\right\}$ is an absolutely continuous statistical structure.

The above examples show that, given an observation, one can change a statistical structure by discarding some impossible values of the parameter, and then, changing the measures, one obtains different statistical structures.

Let $\{E, S\}$ be a measurable space, let $\left\{\mu_{i}, i \in I\right\}$ be a family of probability measures in $S$. Assume that the set of parameters $I$ is equipped with the minimal $\sigma$-algebra $B(I)$ containing all finite subsets of $I$.

Definition 1. We say that

$$
\left\{E, S, \mu_{i}, i \in I\right\}
$$

is a statistical structure if the set of parameters $I$ is equipped with the minimal $\sigma$-algebra $B(I)$ such that all the functions $i \rightarrow \mu_{i}(A), A \in S$, are measurable with respect to $B(I)$.

Definition 2. A statistical structure $\left\{E, S, \mu_{i}, i \in I\right\}$ is called orthogonal (singular) if the measures $\left\{\mu_{i}, i \in I\right\}$ are pairwise orthogonal; that is,

$$
(\forall i)(\forall j)\left(i \in I \& j \in I \& i \neq j \longrightarrow \mu_{i} \perp \mu_{j}\right) .
$$

Definition 3. A statistical structure $\left\{E, S, \mu_{i}, i \in I\right\}$ is called weakly separable if there exists a family of $S$-measurable sets $\left\{X_{i}, i \in I\right\}$ such that

$$
\mu_{i}\left(X_{j}\right)= \begin{cases}1, & i=j \\ 0, & i \neq j\end{cases}
$$

Definition 4. A statistical structure $\left\{E, S, \mu_{i}, i \in I\right\}$ is called separable if there exists a family of $S$-measurable sets $\left\{X_{i}, i \in I\right\}$ such that

1) $\operatorname{card}\left(X_{i} \cap X_{j}\right)<2^{\aleph_{0}}$ for all $i \neq j$,

2) $\mu_{i}\left(x_{j}\right)= \begin{cases}1, & i=j, \\ 0, & i \neq j,\end{cases}$

where $\aleph_{0}$ denotes the cardinality of a countable set.

Definition 5. A statistical structure $\left\{E, S, \mu_{i}, i \in I\right\}$ is called strongly separable if there exists a family of disjoint $S$-measurable sets $\left\{X_{i}, i \in I\right\}$ such that

$$
\mu_{i}\left(X_{i}\right)=1
$$

for all $i \in I$.

Remark 1 . The strong separability of a statistical structure implies its weak separability. Also, the weak separability implies orthogonality (the converse is not true). 
Example 3. Let $E=[0,1] \times[0,1]$ be the unit square and let $S$ be the $\sigma$-algebra of the Borel sets of $E$. Consider the sets

$$
X_{i}=\{0 \leq x \leq 1, y=i, i \in[0,1]\}
$$

and linear Lebesgue measures $\mu_{i}$ in $X_{i}, i \in[0,1]$. Then $\left\{[0,1] \times[0,1], S, \mu_{i}, i \in[0,1]\right\}$ is a strongly separable statistical structure.

Example 4. Let $E=[0,1] \times[0,1]$ be the unit square and let $S$ be the $\sigma$-algebra of the Borel sets of $E$. Consider the sets

$$
X_{i}= \begin{cases}0 \leq x \leq 1, y=i, & i \in[0,1], \\ x=i-2,0 \leq y \leq 1, & i \in[2,3]\end{cases}
$$

and let $\mu_{i}$ be the Lebesgue linear measures in $X_{i}, i \in[0,1] \cup[2,3]$. Then

$$
\left\{[0,1] \times[0,1], S, \mu_{i}, i \in[0,1] \cup[2,3]\right\}
$$

is a separable statistical structure. This structure is not strongly separable.

Example 5. Let $E=[0,1] \times[0,1] \times[0,1]$ be the unit cube and let $S$ be the $\sigma$-algebra of the Borel sets of $E$. Consider the sets

$$
X_{i}= \begin{cases}0 \leq x \leq 1,0 \leq y \leq 1, z=i, & i \in[0,1], \\ x=i-2,0 \leq y \leq 1,0 \leq z \leq 1, & i \in[2,3], \\ 0 \leq x \leq 1, y=i-4,0 \leq z \leq 1, & i \in[4,5],\end{cases}
$$

and let $\mu_{i}$ be the plane Lebesgue measures in $X_{i}, i \in[0,1] \cup[2,3] \cup[4,5]$. Then

$$
\left\{[0,1] \times[0,1] \times[0,1], S, \mu_{i}, i \in[0,1] \cup[2,3] \cup[4,5]\right\}
$$

is a weakly separable statistical structure. Note that this structure is not separable.

Example 6. Let $E=[0,1] \times[0,1] \times[0,1]$ be the unit cube and let $S$ be the $\sigma$-algebra of Borel sets in $E$. Consider the sets

$$
X_{i}= \begin{cases}0 \leq x \leq 1,0 \leq y \leq 1, z=i, & i \in(0,1], \\ x=i-2,0 \leq y \leq 1,0 \leq z \leq 1, & i \in[2,3], \\ 0 \leq x \leq 1, y=i-4,0 \leq z \leq 1, & i \in[4,5]\end{cases}
$$

and let $\mu_{i}$ be the plane Lebesgue measures in $X_{i}, i \in(0,1] \cup[2,3] \cup[4,5]$, while $\mu_{0}$ is the Lebesgue measure in $E$. Then

$$
\left\{E, S, \mu_{i}, i \in[0,1] \times[2,3] \times[4,5]\right\}
$$

is an orthogonal statistical structure. Note that this structure is not strongly separable.

Theorem 1. If a statistical structure $\left\{E, S, \mu_{k}, k=1,2, \ldots\right\}$ is separable or weakly separable, then it is strongly separable.

Proof. Since the statistical structure is separable or weakly separable, there exists a family of $S$-measurable sets $\bar{A}_{1}, \bar{A}_{2}, \ldots$, such that

$$
\mu_{i}\left(\bar{A}_{j}\right)= \begin{cases}1, & i=j \\ 0, & i \neq j\end{cases}
$$


Introduce the sets

$$
\begin{aligned}
& A_{1}=\bar{A}_{1}-\bar{A}_{1} \cap\left(\bigcup_{k \neq 1} \bar{A}_{k}\right), \\
& A_{2}=\bar{A}_{2}-\bar{A}_{2} \cap\left(\bigcup_{k \neq 2} \bar{A}_{k}\right), \\
& \ldots \ldots \ldots \ldots \ldots \ldots \ldots \ldots \ldots \ldots \ldots \ldots \\
& A_{n}=\bar{A}_{n}-\bar{A}_{n} \cap\left(\bigcup_{k \neq n} \bar{A}_{k}\right),
\end{aligned}
$$

It is clear that the sets $A_{1}, A_{2}, \ldots, A_{n}, \ldots$, are mutually disjoint and $\mu_{i}\left(A_{i}\right)=1$ for all $i=1,2, \ldots$. The theorem is proved.

Theorem 2. If a statistical structure $\left\{E, S, \mu_{k}, k=1,2, \ldots\right\}$ is orthogonal, then it is strongly separable.

Proof. The orthogonality implies that there exist $S$-measurable sets $\left\{\widetilde{C}_{i k}\right\}$ such that

$$
\mu_{k}\left(\widetilde{C}_{i k}\right)=0 \text { and } \mu_{i}\left(E-\widetilde{C}_{i k}\right)=0 .
$$

Consider the sets

$$
C_{i}=\bigcup_{k \neq i}\left(E-\widetilde{C}_{i k}\right)
$$

Then

$$
\mu_{i}\left(C_{i}\right)=\mu_{i}\left[\bigcup_{k \neq i}\left(E-\widetilde{C}_{i k}\right)\right] \leq \sum_{k \neq i} \mu_{i}\left(E-\widetilde{C}_{i k}\right)=0
$$

and $\mu_{i}\left(E-C_{i}\right)=1$ for all $i=1,2, \ldots$.

On the other hand,

$$
\mu_{k}\left(E-C_{i}\right)=\mu_{k}\left[E-\bigcup_{k \neq i}\left(E-\widetilde{C}_{i k}\right)\right]=\mu_{k}\left(\bigcup_{k \neq i} C_{i k}\right)=0 \quad \text { if } k \neq i .
$$

This statistical structure is weakly separable and, according to Theorem 1, it is strongly separable. The theorem is proved.

Definition 6. Let $M=\left\{E, S, \mu_{i}, i \in I\right\}$ and $N=\left\{E, S, P_{\alpha}, \alpha \in A\right\}$ be two statistical structures. The statistical structure

$$
M \cup N=\left\{E, S, \mu_{i}, i \in I, P_{\alpha}, \alpha \in A\right\}
$$

is called the sum of $M$ and $N$.

Definition 7. Let $M=\left\{E, S, \mu_{i}, i \in I\right\}$ and $N=\left\{E, S, P_{\alpha}, \alpha \in A\right\}$ be two statistical structures. We say that $M$ is subordinated to $N$ if for every $i \in I$ there exist a sequence $\left\{\alpha_{k}\right\}, \alpha_{k} \in A$, and a sequence $\left\{\rho_{k}\right\}$ such that $\rho_{k} \geq 0, \sum_{k=1}^{\infty} \rho_{k}<\infty$, and the measure $\mu_{i}$ is absolutely continuous with respect to the measure $\sum_{k=1}^{\infty} \rho_{k} P_{\alpha_{k}}$.

In what follows we assume that $\mu_{i}$ considered as functions of the argument $i$ are $B(I)$-measurable.

Definition 8. A probability measure $\mu$ is called the mixture for the statistical structure $\left\{E, S, \mu_{i}, i \in I\right\}$ if there exist a $S \times B(I)$-measurable nonnegative function $\rho(x, i)$ and a probability measure $\gamma$ in $B(I)$ such that

$$
\mu(A)=\int_{I} \int_{A} \rho(x, i) \mu_{i}(d x) \gamma(d i)
$$


for all $A \in S$, and

$$
\int_{I} \int_{E} \rho(x, i) \mu_{i}(d x) \gamma(d i)=1
$$

Definition 9. A statistical structure $M=\left\{E, S, \mu_{i}, i \in I\right\}$ is called pure if none of the measures of $M$ is a mixture of other measures of this family; in other words, an arbitrary measure $\mu_{i_{0}}$ of this family is not the mixture for the statistical structure

$$
\left\{E, S, \mu_{i}, i \in I-\left\{i_{0}\right\}\right\} \text {. }
$$

Definition 10. We say that a statistical structure $M=\left\{E, S, \mu_{i}, i \in I\right\}$ is weakly subordinated to a statistical structure $N=\left\{E, S, P_{\alpha}, \alpha \in A\right\}$ if every measure $\mu_{i}$ is the mixture of the measures $\left\{P_{\alpha}, \alpha \in A\right\}$.

Definition 11. A statistical structure $\left\{E, S, P_{\alpha}, \alpha \in A\right\}$ is called reducible if it is subordinated to some pure statistical structure.

Definition 12. A statistical structure $\left\{E, S, P_{\alpha}, \alpha \in A\right\}$ is called totally reducible if every measure of the statistical structure is a mixture of all other measures of this structure.

In the sequel, we denote by (ZFC) the Zermelo-Fraenkel set theory which includes the axiom of choice (see, for example, [3]).

Theorem 3. In the theory $(Z F C)$, an arbitrary statistical structure $\left\{E, S \mu_{b}, b \in B\right\}$ either is subordinated to a certain statistical orthogonal structure or is totally irreducible.

Proof. It is known that one can introduce an orthogonal statistical structure in every set $E$ whose cardinality is a continuum ( $\operatorname{card} E=c$ ) in such a way that the cardinality of the structure is maximal; that is, the cardinality is $2^{2^{c}}$ (see [4]). In the theory (ZFC), an arbitrary set can be well ordered (see [3]). Let $I$ be well ordered. We construct the pairwise orthogonal measures $\eta_{\alpha}$ with the help of transfinite induction.

Let $\eta_{1}=\mu_{i_{1}}$ for the first element of $I$ and let pairwise orthogonal measures $\eta_{\gamma}$ be defined for all ordinals $\gamma$ that are less than $\alpha$. This means that, for any measure $\mu_{\gamma}$, there exists a countable sequence $\left\{\gamma_{k}\right\}$ and numbers $\rho_{k} \geq 0$ such that $\sum_{k=1}^{\infty} \rho_{k}<\infty$ and the measure $\mu_{\gamma}$ is absolutely continuous with respect to the measure $\sum_{k=1}^{\infty} \rho_{k} \eta_{\gamma_{k}}$. Thus a countable family of pairwise orthogonal probability measures $\eta_{\gamma}, \gamma<\alpha$, is defined. Then, for $\mu_{\gamma}$, there is at most a countable set $\Gamma_{\alpha}$ of $\gamma<\alpha$ such that $\eta_{\gamma}$ is not orthogonal to $\mu_{\alpha}$. Set

$$
\widehat{\eta}_{\alpha}(A)=\mu_{\alpha}(A)-\sum_{\gamma \in \Gamma_{\alpha}} \int_{A} \frac{d \mu_{\alpha}}{d \eta_{\gamma}}(x) \eta_{\gamma}(d x)
$$

for all $A \in S$.

Since the statistical structure $\left\{E, S, \eta_{\gamma}, \gamma \in \Gamma_{\alpha}\right\}$ is orthogonal and card $\Gamma_{\alpha} \leq \aleph_{0}$, Theorem 2 implies that the structure is strongly separable. In other words, there are mutually disjoint $S$-measurable sets $A_{\gamma_{i}}, i=1,2, \ldots$, such that

$$
\eta_{\gamma_{i}}\left(A_{j_{i}}\right)=1, \quad \forall i=1,2, \ldots,
$$

whence

$$
\sum_{\gamma \in \Gamma_{\alpha}} \int_{A} \frac{d \mu_{\alpha}}{d \eta_{\gamma}} d \eta_{\gamma}=\int_{A \cap A_{1}} \frac{d \mu_{\alpha}}{d \eta_{\gamma_{1}}} d \eta_{\gamma_{1}}+\int_{A \cap A_{2}} \frac{d \mu_{\alpha}}{d \eta_{\gamma_{2}}} d \eta_{\gamma_{2}}+\cdots \leq \mu_{\alpha}(A)
$$

and

$$
\mu_{\alpha}(A)=\sum_{\gamma \in \Gamma_{\alpha}} \int_{A} \frac{d \mu_{\alpha}}{d \eta_{\gamma}} d \eta_{\gamma}+\widehat{\eta}_{\alpha}(A)
$$


Put

$$
\eta_{\alpha}= \begin{cases}0, & \widehat{\eta}_{\alpha}(E)=0 \\ \frac{\widehat{\eta}_{\alpha}(A)}{\widehat{\eta}_{\alpha}(E)}, & \widehat{\eta}_{\alpha}(E)>0 .\end{cases}
$$

Let $A_{1}=\left\{\alpha: \widehat{\eta}_{\alpha}(E)>0\right\}$. Then every probability measure $\mu_{\alpha}$ is subordinated to an orthogonal statistical structure $\left\{E, S, \eta_{\alpha}, \alpha \in A_{1}\right\}$. If $\widehat{\eta}_{\alpha}(A)=0$ for all $\alpha$, then every measure $\mu_{\alpha}$ is a mixture of all other measures $\mu_{\gamma}, \gamma \neq \alpha$. This means that the statistical structure is totally irreducible. Theorem 3 is proved.

Example 7. Let $\Theta$ consist of two points $\theta_{1}$ and $\theta_{2}$ and let two probability measures $\mu_{\theta_{1}}$ and $\mu_{\theta_{2}}$ be given in $(E, S)$. One can always find three mutually disjoint sets $A_{1}, A_{2}$, and $A_{3}$ such that $E=A_{1} \cup A_{2} \cup A_{3}$ and that the measures $\mu_{\theta_{1}}$ and $\mu_{\theta_{2}}$ are equivalent in the set $A_{2}, \mu_{\theta_{2}}\left(A_{1}\right)=0$, and $\mu_{\theta_{1}}\left(A_{3}\right)=0$. Let

$$
\nu_{1}(A)=\frac{\mu_{\theta_{1}}\left(A \cap A_{1}\right)}{\mu_{\theta_{1}}\left(A_{1}\right)}, \quad \nu_{2}(A)=\frac{\mu_{\theta_{2}}\left(A \cap A_{3}\right)}{\mu_{\theta_{2}}\left(A_{3}\right)}, \quad \nu_{3}(A)=\frac{\mu_{\theta_{1}}\left(A \cap A_{2}\right)}{\mu_{\theta_{1}}\left(A_{2}\right)}
$$

for all sets $A \in S$. We are going to show that the statistical structure $\left\{E, S, \mu_{\theta_{1}}, \mu_{\theta_{2}}\right\}$ is subordinated to the orthogonal statistical structure $\left\{E, S, \nu_{1}, \nu_{2}, \nu_{3}\right\}$. Indeed, for all sets $A \in S$, we have

$$
\begin{aligned}
\mu_{\theta_{1}}(A) & =\mu_{\theta_{1}}\left(A \cap A_{1}\right)+\mu_{\theta_{1}}\left(A \cap A_{2}\right)+\mu_{\theta_{1}}\left(A \cap A_{3}\right) \\
& =\frac{\mu_{\theta_{1}}\left(A \cap A_{1}\right)}{\mu_{\theta_{1}}\left(A_{1}\right)} \mu_{\theta_{1}}\left(A_{1}\right)+\frac{\mu_{\theta_{1}}\left(A \cap A_{2}\right)}{\mu_{\theta_{1}}\left(A_{2}\right)} \mu_{\theta_{1}}\left(A_{2}\right)=\alpha \nu_{1}+\beta \nu_{3},
\end{aligned}
$$

where $\alpha=\mu_{\theta_{1}}\left(A_{1}\right), \beta=\mu_{\theta_{1}}\left(A_{2}\right)$; and

$$
\begin{aligned}
\mu_{\theta_{2}}(A) & =\mu_{\theta_{2}}\left(A \cap A_{1}\right)+\mu_{\theta_{2}}\left(A \cap A_{2}\right)+\mu_{\theta_{2}}\left(A \cap A_{3}\right) \\
& =\frac{\mu_{\theta_{2}}\left(A \cap A_{3}\right)}{\mu_{\theta_{2}}\left(A_{3}\right)} \mu_{\theta_{2}}\left(A_{3}\right)+\frac{\mu_{\theta_{2}}\left(A \cap A_{2}\right)}{\mu_{\theta_{1}}\left(A_{2} \cap A_{2}\right)} \frac{\mu_{\theta_{1}}\left(A \cap A_{2}\right)}{\mu_{\theta_{1}}\left(A \cap A_{2}\right)} \mu_{\theta_{1}}\left(A_{2} \cap A_{2}\right) \\
& =\alpha_{1} \nu_{2}+\beta_{2} \nu_{3},
\end{aligned}
$$

where

$$
\alpha_{1}=\mu_{\theta_{2}}\left(A_{3}\right), \quad \beta_{2}=\mu_{\theta_{2}}\left(A \cap A_{2}\right) \frac{\mu_{\theta_{1}}\left(A_{2}\right)}{\mu_{\theta_{1}}\left(A \cap A_{2}\right)} .
$$

Thus the statistical structure $\left\{E, S, \mu_{\theta_{1}}, \mu_{\theta_{2}}\right\}$ is subordinated to the orthogonal statistical structure $\left\{E, S, \nu_{1}, \nu_{2}, \nu_{3}\right\}$.

Example 8. Let $E=[0,1] \times[0,1]$ be the unit square and let $S$ be the $\sigma$-algebra of Borel sets in $E$. Consider the sets

$$
X_{i}= \begin{cases}0 \leq x \leq 1, y=i, & i \in[0,1], \\ x=i-2,0 \leq y \leq 1, & i \in[2,3]\end{cases}
$$

and the linear Lebesgue measures $\mu_{i}$ in these sets:

$$
\mu_{i}\left(X_{j}\right)= \begin{cases}1, & i=j \\ 0, & i \neq j\end{cases}
$$

Then we consider the following plane measure:

$$
\nu_{1}(A)=\int_{0}^{1} \mu_{i}\left(A \cap X_{i}\right) \gamma_{1}(d i)=\int_{0}^{1} \int_{A} \mu_{i}(d x) \gamma_{1}(d i),
$$

where $\gamma_{1}$ is the Lebesgue measure in $[0,1]$. It is clear that $\nu_{1}$ is a mixture of the measures $\left\{\mu_{i}, i \in[0,1]\right\}$. Let

$$
\nu_{2}(A)=\int_{2}^{3} \mu_{i}\left(A \cap X_{i}\right) \gamma_{2}(d i)=\int_{2}^{3} \int_{A} \mu_{i}(d x) \gamma_{2}(d i),
$$


where $\gamma_{2}$ is the Lebesgue measure in $[2,3]$. It is clear that $\nu_{2}$ is a mixture of the measures $\left\{\mu_{i}, i \in[2,3]\right\}$.

For a fixed measure $\mu_{i_{0}}$, put

$$
q_{i_{0}}=\sum_{\nu \in N} \int \frac{d \mu_{i_{0}}}{d \nu}(x) \nu(d x)
$$

where the supremum is considered with respect to all probability measures $\nu$ belonging to the set $E$. We say that a family $N$ of probability measures is countably convex if

$$
\sum_{k=1}^{\infty} \alpha_{k} \nu_{k} \in N
$$

for $\nu_{k} \in N$, where the numbers $\left\{\alpha_{k}\right\}$ are such that $\alpha_{k}>0$ and $\sum_{k=1}^{\infty} \alpha_{k}=1$.

Lemma 1. If a family $N$ is countably convex, then $q_{i_{0}}$ is attained.

Proof. It is clear that the result is true if $q_{i_{0}}=0$. Assume that $0<q<1$ and that, for all $k$, there is a measure $\nu_{k} \in N$ such that

$$
q_{i_{0}}-\frac{1}{k}<\int_{E} \frac{d \mu_{i_{0}}}{d \nu_{k}}(x) d \nu_{k}(x) \leq q_{i_{0}} .
$$

Let

$$
\bar{\nu}=\sum_{k=1}^{\infty} \alpha_{k} \nu_{k}, \quad \text { where } \sum_{k=1}^{\infty} \alpha_{k}=1 \quad \text { and } \alpha_{k}>0 .
$$

We represent the measures $\bar{\nu}$ and $\mu$ in terms of $\nu_{k}$ as follows:

$$
\bar{\nu}=\beta \nu_{k}+\nu, \quad \mu_{i_{0}}=\delta \nu_{k}+\nu^{\prime},
$$

where $\beta>0$ and $\delta>0$.

It is clear that $d \bar{\nu} / d \nu_{k}=\beta$ and $d \mu_{i_{0}} / d \nu_{k}=\delta$. Then

$$
\frac{d \mu_{i_{0}}}{d \bar{\nu}}=\frac{d\left(\delta \nu_{k}+\nu^{\prime}\right)}{d \bar{\nu}}=\delta \frac{d \nu_{k}}{d \bar{\nu}}+\frac{d \nu^{\prime}}{d \bar{\nu}}
$$

Multiplying the latter equality by $d \bar{\nu} / d \nu_{k}$ we obtain

$$
\frac{d \mu_{i_{0}}}{d \bar{\nu}} \frac{d \bar{\nu}}{d \nu_{k}}=\delta \frac{d \nu_{k}}{d \bar{\nu}} \frac{d \bar{\nu}}{d \nu_{k}}+\frac{d \nu^{\prime}}{d \bar{\nu}} \frac{d \bar{\nu}}{d \nu_{k}}=\delta=\frac{d \mu_{i_{0}}}{d \nu_{k}} .
$$

Thus

It is clear that

$$
\frac{d \mu_{i_{0}}}{d \mu_{k}}=\frac{d \mu_{i_{0}}}{d \bar{\nu}} \frac{d \bar{\nu}}{d \nu_{k}}
$$

$$
q-\frac{1}{k}<\int_{E} \frac{d \mu_{i_{0}}}{d \nu_{k}} d \nu_{k}=\int_{E} \frac{d \mu_{i_{0}}}{d \bar{\nu}} \frac{d \bar{\nu}}{d \nu_{k}} \frac{d \nu_{k}}{d \bar{\nu}} d \bar{\nu} \leq \int_{E} \frac{d \mu_{i_{0}}}{d \bar{\nu}} d \bar{\nu}
$$

that is,

$$
q-\frac{1}{k} \leq \int_{E} \frac{d \mu_{i_{0}}}{d \bar{\nu}} d \bar{\nu} \leq q
$$

for all $k>0$. Passing to the limit as $k \rightarrow \infty$,

$$
q=\int_{E} \frac{d \mu_{i_{0}}}{d \bar{\nu}} d \bar{\nu}
$$

Hence $\bar{\nu} \in N$. The lemma is proved. 
Theorem 4. For every orthogonal statistical structure

$$
M=\left\{E, S, \mu_{b}, b \in B\right\},
$$

there exist three statistical structures $N_{1}, N_{2}$, and $N_{3}$ such that $N_{1}$ is pure, $N_{2}$ is weakly subordinated to $N_{1}, N_{3}$ is totally irreducible, and $M$ is subordinated to $N_{1} \cup N_{2} \cup N_{3}$.

Proof. Put

$$
q_{b_{0}}=\sup \int_{E} \frac{d \mu_{b_{0}}}{d \nu}(x) d \nu(x)
$$

where sup is considered with respect to probability measures $\nu$ being mixtures of measures $\nu_{b}, b \neq b_{0}$.

For every $k$, there exists $\nu_{k, b_{0}}$ such that

$$
q_{b_{0}}-\frac{1}{k}<\int_{E} \frac{d \mu_{b_{0}}}{d \nu_{k, b_{0}}}(x) d \nu_{k, b_{0}}(x) \leq q_{b_{0}}
$$

where

$$
\nu_{k, b_{0}}(A)=\int_{B-\left(b_{0}\right)} \int_{A} \rho_{k}(x, b) \mu_{b}(d x) \gamma_{k}(d b) .
$$

Choose numbers $\beta_{k}>0$ such that $\sum_{k=1}^{\infty} \beta_{k}=1$ and put $\bar{\gamma}=\sum_{k=1}^{\infty} \beta_{k} \gamma_{k}$. Then $\gamma_{k}$ is absolutely continuous with respect to $\bar{\gamma}$ and

$$
\nu_{k, b_{0}}(A)=\int_{B-\left(b_{0}\right)} \int_{A} \rho_{k}(x, b) \mu_{b}(d x) \frac{d \gamma_{k}}{d \delta}(b) \bar{\gamma}(d b) .
$$

Put

Then

$$
\widetilde{\rho}_{k}(x, b)=\rho_{k}(x, b) \frac{d \gamma_{k}}{d \delta}(b)
$$

$$
\nu_{k, b_{0}}(A)=\int_{B-\left(b_{0}\right)} \int_{A} \widetilde{\rho}_{k}(x, b) \mu_{b}(d x) \bar{\gamma}_{k}(d b) .
$$

Let the numbers $\alpha_{k}>0$ be such that $\sum_{k=1}^{\infty} \alpha_{k}=1$. Put

$$
\nu_{b_{0}}=\sum_{k=1}^{\infty} \alpha_{k} \nu_{k, b_{0}} \text {. }
$$

Then

$$
\begin{aligned}
\alpha_{b_{0}}(A) & =\sum_{k=1}^{\infty} \alpha_{k} \nu_{k, b_{0}}(A)=\sum_{k=1}^{\infty} \alpha_{k} \int_{B-\left(b_{0}\right)} \int_{A} \widetilde{\rho}_{k}(x, b) \mu_{A}(d x) \bar{\gamma}(d b) \\
& =\int_{B-\left(b_{0}\right)} \int_{A}\left[\sum_{k=1}^{\infty} \alpha_{k} \widetilde{\rho}_{k}(x, b)\right] \mu_{B}(d x) \bar{\gamma}(d b) .
\end{aligned}
$$

Let $\widetilde{\rho}(x, b)=\sum_{k=1}^{\infty} \alpha_{k} \widetilde{\rho}_{k}(x, b)$. Then

$$
\nu_{b_{0}}(A)=\int_{B-\left(b_{0}\right)} \int_{A} \tilde{\rho}(x, b) \mu_{B}(d x) \bar{\gamma}(d b) .
$$

According to Lemma 1 , the supremum is attained at $\nu_{b_{0}}$. In other words,

$$
q_{b_{0}}=\int_{E} \frac{d \mu_{b_{0}}}{d \nu_{b_{0}}}(x) \nu_{b_{0}}(d x)
$$

For $q_{b_{0}}<1$, put

$$
\bar{\mu}_{b_{0}}(A)=\frac{1}{1-q_{b_{0}}}\left[\mu_{b_{0}}(A)-\int_{A} \frac{d \mu_{b_{0}}}{d \nu_{b_{0}}}(x) d \nu_{b_{0}}(x)\right] .
$$


Let $B_{1}$ be the set of those $b_{0}$ for which $q_{b_{0}}<1$. Then the structure

$$
N_{1}=\left\{E, S, \bar{\mu}_{b}, b \in B_{1}\right\}
$$

is pure; that is, $N_{1}$ is reducible.

Consider the following statistical structure:

$$
D=\left\{E, S, \widetilde{\mu}_{b}, b \in B_{2}\right\},
$$

where

$$
\widetilde{\mu}_{b}(A)=\frac{1}{q_{b}} \int_{A} \frac{d \mu_{b}}{d \nu_{b}}(x) d \nu_{b}(x)
$$

and where $B_{2}$ is the set of those $b$ for which $q_{b}>0$. Put

$$
\bar{q}_{b_{0}}=\sup \int_{E} \frac{d \widetilde{\mu}}{d \bar{\nu}} d \bar{\nu}
$$

where sup is evaluated with respect to the measures $\bar{\nu}$ being mixtures of $\bar{\mu}_{b}, b \neq b_{0}$. According to Lemma 1, sup is attained. Assume that $\bar{\nu}_{b_{0}}$ is such that

$$
\bar{q}_{b_{0}}=\int_{E} \frac{d \bar{\mu}}{d \bar{\nu}_{b_{0}}} d \bar{\nu}_{b_{0}}(d x)
$$

where

Put

$$
\bar{\nu}_{b_{0}}(A)=\int_{B-\left\{b_{0}\right\}} \int_{A} \bar{\rho}(x, b) \widetilde{\mu}_{b}(d x) \bar{\gamma}(d b) .
$$

if $\bar{q}_{b_{0}}<1$, and

$$
\widetilde{\bar{\mu}}_{b_{0}}(A)=\frac{1}{1-\bar{q}_{b_{0}}}\left[\widetilde{\mu}_{b_{0}}(A)-\int_{A} \frac{d \widetilde{\mu}_{b_{0}}}{d \bar{\nu}_{b_{0}}}(x) d \bar{\nu}_{b_{0}}(x)\right]
$$

$$
\overline{\bar{\mu}}_{b_{0}}(A)=\frac{1}{\bar{q}_{b_{0}}} \int_{A} \frac{d \widetilde{\mu}_{b_{0}}}{d \bar{\nu}_{b_{0}}}(x) d \bar{\nu}_{b_{0}}(x)
$$

if $\bar{q}_{b_{0}}>0$. Let $B_{3}$ be the set of those $b_{0}$ for which $\bar{q}_{b_{0}}<1$. Accordingly, let $B_{4}$ be the set of those $b_{0}$ for which $\bar{q}_{b_{0}}>0$. It is clear that the structure $\left\{E, S, \widetilde{\mu}_{b}, b \in B_{2}\right\}$ is subordinated to the structure $\left\{E, S, \widetilde{\bar{\mu}}_{b}, b \in B_{3}, \overline{\bar{\mu}}_{b}, b \in B_{4}\right\}$ and the structure $N_{2}=$ $\left\{E, S, \overline{\bar{\mu}}_{b}, b \in B_{4}\right\}$ is weakly subordinated to the structure $N_{1}=\left\{E, S, \bar{\mu}_{b}, b \in B_{1}\right\}$, while the structure $N_{3}=\left\{E, S, \widetilde{\bar{\mu}}_{b}, b \in B_{3}\right\}$ is totally irreducible. It is clear that the structure $M$ is subordinated to the sum $N_{1} \cup N_{2} \cup N_{3}$ of the three structures. The theorem is proved.

\section{BIBLIOGRAPHY}

1. I. M. Ibramkhalilov and A. V. Skorokhod, Consistent Estimators of Parameters of Random Processes, "Naukova Dumka", Kiev, 1980. (Russian) MR.584624 (82h:62140)

2. Z. S. Zerakidze, A Hilbert space of measures, Ukr. Math. J. 38 (1986), no. 2, 148-154. MR841043 (88f:46062)

3. F. R. Drake, Set Theory: An Introduction to Large Cardinals, North-Holland Publishing Company, Amsterdam, 1974.

4. Z. S. Zerakidze, Weakly separable and separable families of probability measures, Soobshch. Akad. Nauk Gruzin. SSR 113 (1984), no. 2, 273-275. (Russian) MR780715 (86e:28010)

Gori State University, Chavchavadze Street, 53, Gori, Georgia

E-mail address: z.zerakidze@gmail.ru

Ilia Vekua Institute of Applied Mathematics, University Street 3, Tbilisi, 0186, Georgia

E-mail address: giasokhi1@i.ua 